https://doi.org/10.22319/rmcp.v10i4.4470

Artículo

\title{
Caracterización productiva y socioeconómica del sistema de producción ovina, en un área natural protegida de México
}

Daniel Hernández Valenzuela ${ }^{\text {a }}$

Ernesto Sánchez Vera ${ }^{b} *$

William Gómez Demetrio ${ }^{b}$

Carlos Galdino Martínez García b

${ }^{a}$ Universidad Autónoma de Guerrero, Facultad de Ciencias Agropecuarias y Ambientales, Guerrero, México.

${ }^{\mathrm{b}}$ Universidad Autónoma del Estado México, Instituto de Ciencias Agropecuarias y Rurales, Estado de México, México. Campus "El Cerrillo Piedras Blancas", 50090, Toluca, Estado de México. México.

* Autor de correspondencia: esanchezv@uaemex.mx

\section{Resumen:}

Ante el incremento de la presencia humana y sus actividades productivas en áreas naturales protegidas, se planteó el objetivo de caracterizar el sistema de producción ovina, en el Nevado de Toluca, con información agrícola, socioeconómica y uso de los recursos de pastoreo. Un total de 162 productores fueron entrevistados, se analizaron 25 variables con estadística multivariada y univariada. Mediante análisis de componentes principales se obtuvieron seis factores que explican el $71 \%$ de la varianza. El análisis cluster permitió identificar tres grupos de productores: pequeños $(28 \%)$, intermedios $(35 \%)$ y capitalizados $(6 \%)$, diferenciados por el número de animales, superficie cultivada e ingresos $(P<0.05)$. En el sistema de producción, se observó alta mortalidad de corderos $(23 \%)$, la siembra de avena (Avena sativa) en el $50 \%$ de la superficie y un porcentaje variable de superficie, sembrada de maíz (Zea mays). La edad y escolaridad fueron similares entre grupos $(P>0.05)$ y se encontró que los rebaños aportan menos del $30 \%$ del ingreso familiar. El $58 \%$ de los rebaños 
ingresa al bosque, para realizar pastoreo circulante, pero el $60 \%$ se maneja semi-estabulado. Se concluye que la producción del sistema no depende de la capitalización del productor, pero el rebaño es esencial en la economía familiar, y el manejo de los animales es compatible con los esfuerzos de conservación del área natural protegida.

Palabras clave: Pequeños rumiantes, Pastoreo circulante, Nevado de Toluca, Recursos naturales, Silvopastoril.

Recibido: 25/04/2017

Aceptado: 30/08/2018

\section{Introducción}

Los sistemas ganaderos ocupan alrededor del $45 \%$ de la superficie terrestre ${ }^{(1)}$ y tienen muchas variantes, desde pastoreo extensivo y mixtos, que integran la producción agrícola y ganadera, hasta aquellos altamente tecnificados, orientados al mercado ${ }^{(2)}$. Los sistemas extensivos de pequeños rumiantes suelen pastorear en áreas de vegetación nativa ${ }^{(3)}$, en una intrincada relación entre agricultura, ganadería y recursos naturales, de la cual dependen muchos hogares ${ }^{(4)}$. Esta relación es debatida, en torno a los impactos ambientales de la ganadería $^{(5)}$ y cobra mayor importancia en áreas naturales protegidas (ANP), donde las personas suelen pastorear sus animales, lo que plantea un dilema entre el aprovechamiento y la restricción en el uso de los recursos naturales ${ }^{(4)}$.

Los sistemas de producción, entendidos como la población de unidades similares en su base de recursos, medios de vida y limitaciones ${ }^{(6)}$, pueden ser caracterizados a través de sus componentes estructurales, indicadores técnico-productivos y económicos. Esto permite integrar elementos complejos y diversos en su análisis ${ }^{(7)}$, además de soportar estrategias y recomendaciones orientadas a lograr una mayor eficiencia y rentabilidad en la producción. Este es el caso de la caracterización de la ganadería en áreas protegidas ${ }^{(8)}$, en las que se incorpora la actividad pastoral como principal elemento de interacción entre el ganado y los recursos naturales ${ }^{(5,9,10)}$. Sin embargo, en México no existen estudios de sistemas de producción ovina (SPO) en ANP, que incorporen aspectos socioeconómicos, la dinámica de pastoreo y sus resultados productivos. Así, el objetivo de esta investigación fue la caracterización productiva, agrícola y socioeconómica del sistema de producción ovina en el Área Natural Protegida (ANP), Nevado de Toluca (NT), para analizar el uso de los recursos de pastoreo, con lo que se contribuye en la comprensión de este sistema y la identificación de oportunidades para su mejora. 


\section{Material y métodos}

\section{Área de estudio}

La investigación se llevó a cabo en el ANP, Nevado de Toluca, en el Estado de México (México). Se localiza entre los 3,000 y 4,660 msnm, clima semi-frío subhúmedo (CEh), temperatura media anual entre -2 y $7^{\circ} \mathrm{C}$ y precipitación anual de 1,000 a $1,400 \mathrm{~mm}$. Tiene una extensión de 53,987 ha, que pertenece a 61 núcleos agrarios. Aquí se ubican 20 localidades y alrededor de 10,000 habitantes ${ }^{(11)}$, que poseen unas 191,000 cabezas de ganado. De éstas $60 \%$ son ovinos, pastoreados en áreas de vegetación arbórea (bosque) y pastizales, dentro de áreas de uso comunal ${ }^{(11)}$. El Nevado de Toluca fue re-categorizado de Parque Nacional a Área de Protección de Flora y Fauna, en 2013. Esta categoría permite a las personas que habitan el ANP mantener la propiedad de su tierra y realizar algunas actividades productivas, sin cambiar el uso del suelo ${ }^{(12)}$.

\section{Identificación de productores y colecta de información}

Se aplicaron 162 cuestionarios durante el año 2015, a productores de ovinos, elegidos mediante muestreo por conveniencia, como en otros estudios que no se logró construir un marco de muestreo confiable ${ }^{(13)}$. El cuestionario tuvo tres apartados, i) ganadería que rescató el número de animales, alimentación y pastoreo, reproducción, sanidad y prácticas técnicas, ii) actividad agrícola, identificó el número de cultivos, superficie cultivada y maquinaria, iii) características socioeconómicas, se preguntó por edad y nivel de estudios del jefe de familia, fuentes de ingreso, participación de la familia, experiencia y capacitación. Los productores no tuvieron registros de información técnica y económica, lo que es una situación común en los sistemas de producción familiar ${ }^{(7,14,15)}$. Por esto, la información recabada se complementó con observaciones directas a través de visitas a las unidades de producción y además se realizaron 40 entrevistas a profundidad a los pastores, durante sus recorridos de pastoreo.

\section{Análisis estadístico}

Se realizó un análisis de componentes principales (ACP) utilizando 25 variables, para tener una aproximación inicial, a la agrupación de las variables que describen el SPO en esta región. El ACP permitió obtener un grupo reducido de combinaciones lineales (componentes o factores), que explicaron la mayor varianza posible de los datos originales, con mínima pérdida de información ${ }^{(16)}$. La parsimonia de los componentes principales $(\mathrm{CP})$ se verificó con la prueba Kaiser-Meyer-Olkin y la adecuación de la muestra con la prueba de esfericidad de Bartlett $^{(16)}$. Se aplicó la rotación ortogonal varimax a los CP, para mejorar su interpretación $^{(6,16)}$. 
Las combinaciones lineales del ACP se introdujeron al análisis clúster jerárquico (AC) para formar grupos de productores y caracterizar los SPO dentro de ellos. Se empleó el método de Ward para la aglomeración de casos y la distancia euclídea cuadrada como medida de similaridad. El número de grupos se obtuvo del análisis del dendrograma y del coeficiente de aglomeración $^{(6)}$. Las diferencias entre grupos se examinaron con análisis de varianza (variables cuantitativas), mediante la comparación de medias de Hochberg, dado que el tamaño de los grupos no fue homogéneo ${ }^{(17)}$. Las variables categóricas se examinaron con tablas de contingencia y prueba de Ji cuadrada ${ }^{(6)}$. Los resultados del análisis estadístico se triangularon con las observaciones de campo y las entrevistas. Para todos los análisis estadísticos se usó el programa SPSS versión 22.0.

\section{Resultados y discusión}

\section{El sistema de producción ovina en el Nevado de Toluca}

El SPO en el NT es un sistema familiar de baja tecnificación, estrechamente ligado a la agricultura, que cumple funciones de seguridad financiera, semejante al SPO descrito por Vázquez et $a l^{(18)}$. La información mostró que la mayoría de los rebaños son alimentados en pastoreo, con suplementación en corral. Predominan las razas Suffolk, Hampshire y sus cruces, que se eligen por su peso y facilidad de manejo. La reproducción se realiza mediante empadre continuo, pero las hembras muestran una marcada estacionalidad, ya que los apareamientos se concentran en verano, con partos entre noviembre y febrero, que es la época más fría del año, lo que probablemente influye en el aumento de la mortalidad de corderos a causa de enfermedades respiratorias.

\section{Factores que caracterizan los SPO}

El ACP determinó seis componentes principales (CP) que explicaron el $71 \%$ de la varianza. La prueba KMO (0.61) y la esfericidad de Bartlett $(P<0.001)$ confirmaron la confiablidad del análisis. En los CP se retuvo el $60 \%$ de las variables analizadas (Cuadro 1), lo que coincide con análisis como los de Toro-Mujica et $a l^{(7,13)}$, que han retenido alrededor del $64 \%$ de las variables. Esto sugiere que para caracterizar sistemas de producción puede ser suficiente con incluir entre 10 y 20 variables y que la dimensión de la unidad productiva (número de hectáreas y animales), tiene mayor relevancia clasificatoria ${ }^{(19)}$. Se descartaron del ACP las variables con valores de comunalidad menores a 0.5 , porque implica que estas variables tuvieron una baja asociación a los componentes principales seleccionados, como lo han sugerido Hair et al, y Field ${ }^{(16,17)}$. 
Cuadro 1: Componentes principales, con sus variables asociadas, los coeficientes de correlación y la varianza explicada por cada componente

\begin{tabular}{|c|c|c|c|}
\hline Componente & Variables & $\begin{array}{l}\text { Correlación } \\
\text { Variable- } \\
\text { Factor }\end{array}$ & $\begin{array}{l}\text { Varianza } \\
\text { explicada* }\end{array}$ \\
\hline \multirow{3}{*}{1} & Animales & .770 & \multirow{3}{*}{$\begin{array}{l}20.2 \\
(20.2)\end{array}$} \\
\hline & Partos, \% & .768 & \\
\hline & Superficie cultivada, ha & .767 & \\
\hline \multirow{3}{*}{2} & Edad, años & .903 & \multirow{3}{*}{$\begin{array}{l}14.3 \\
(34.5)\end{array}$} \\
\hline & Escolaridad, años & -.712 & \\
\hline & Experiencia, años & 808 & \\
\hline \multirow{2}{*}{3} & Mortalidad de adultos, $\%$ & .746 & 11.2 \\
\hline & Mortalidad de crías, $\%$ & .739 & $(45.7)$ \\
\hline \multirow{3}{*}{4} & Especies cultivadas & .678 & \multirow{3}{*}{$\begin{array}{l}9.4 \\
(55.1)\end{array}$} \\
\hline & Participación familiar, \# & .654 & \\
\hline & Maquinaria, \# & .792 & \\
\hline \multirow{2}{*}{5} & Pastoreo en bosque, $\%$ & .779 & 8.9 \\
\hline & Distancia de pastoreo, $\mathrm{km}$ & .816 & (64) \\
\hline \multirow{2}{*}{6} & Destete, $\%$ & .830 & 6.9 \\
\hline & Desparasitación, \% & .717 & $(71)$ \\
\hline
\end{tabular}

El CP 1 (capitalización) integró variables relativas a los activos del sistema (Cuadro 1), que determinan la capacidad de inversión en prácticas técnicas ${ }^{(20)}$. El CP 2 (capital humano) mostró la relación inversa entre el nivel de estudios del jefe de familia, con su edad y experiencia, que es un reflejo de que los adultos tuvieron menor oportunidad de estudiar, debido en parte, a la falta de oportunidades de empleo formal y nula infraestructura para acceder a servicios educativos, problemática asociada a las características propias del ANP, lo cual derivó en la pronta incorporación a las actividades productivas ${ }^{(21)}$.

El CP 3 (sanidad animal) destacó la mortalidad de animales adultos (11\%) y corderos (23\%), superior a los valores observados por Galaviz-Rodríguez et al ${ }^{(22)}$ en otros SPO, por lo que el manejo sanitario es un área de oportunidad para mejorar la eficiencia productiva y económica del sistema. El CP 4 (agricultura) asoció la diversidad de cultivos con la participación familiar y uso de maquinaria, características de los sistemas mixtos, en los que el aprovechamiento de los recursos agrícolas y ganaderos depende del trabajo familiar ${ }^{(19)}$.

El CP 5 (pastoreo circulante) relacionó el pastoreo dentro del bosque con la distancia recorrida. En este manejo los animales pastan libremente en una superficie durante periodos cortos (alrededor de $10 \mathrm{~min}$ ), en recorridos relativamente largos (2 a $4 \mathrm{~km}$ ), similar al Mediterráneo Francés, donde se realizan circuitos de pastoreo ${ }^{(9)}$. El CP 6 (prácticas técnicas) asoció el destete que ocurre al momento de la venta, sin esquemas de engorda que mejoren 
los ingresos de los productores (14) y la desparasitación que se realiza en el $70 \%$ de los rebaños, superior al $58 \%$ de otros $\mathrm{SPO}^{(23)}$.

\section{Grupos de productores de ovinos en el NT}

Con el análisis clúster se obtuvieron tres grupos de productores, diferenciados principalmente por la cantidad de animales, superficie cultivada y sus ingresos ovinos y agrícolas. Los grupos se clasificaron como productores pequeños (Grupo 1), intermedios (Grupo 2) y capitalizados (Grupo 3), con base en estas diferencias, tal como se ha realizado en otras investigaciones $^{(8,24)}$. A continuación se describen los aspectos productivos, socioeconómicos y uso de los recursos de pastoreo, de acuerdo a los grupos identificados.

\section{Producción ovina}

En el Cuadro 2 se observan las características productivas. Los pequeños productores (28\%) tienen la menor cantidad de animales, destetan y desparasitan en menor porcentaje, probablemente porque reciben menos capacitación. Por otro lado, los productores intermedios $(35 \%)$ se categorizaron así porque poseen una cantidad intermedia de animales; en este grupo se ubica el porcentaje más alto de desparasitación y destete. Mientras que los productores capitalizados $(6 \%)$ tienen la mayor cantidad de animales, sus rebaños son alrededor del $120 \%$, más grandes que en otras regiones de México ${ }^{(18,22)}$, pero un $20 \%$ menores a los rebaños europeos ${ }^{(3,8)}$. A pesar de las diferencias en el nivel de capitalización, el porcentaje de partos y la mortalidad de corderos fueron similares $(P>0.05)$ en los tres grupos, lo que demuestra que la base de recursos no se refleja en la productividad ${ }^{(19)}$, probablemente por falta de capacitación o fallas en la misma. 
Cuadro 2: Características cuantitativas (media y EE) y cualitativas (\%) del sistema de producción ovina por grupos de productores, formados por el análisis clúster

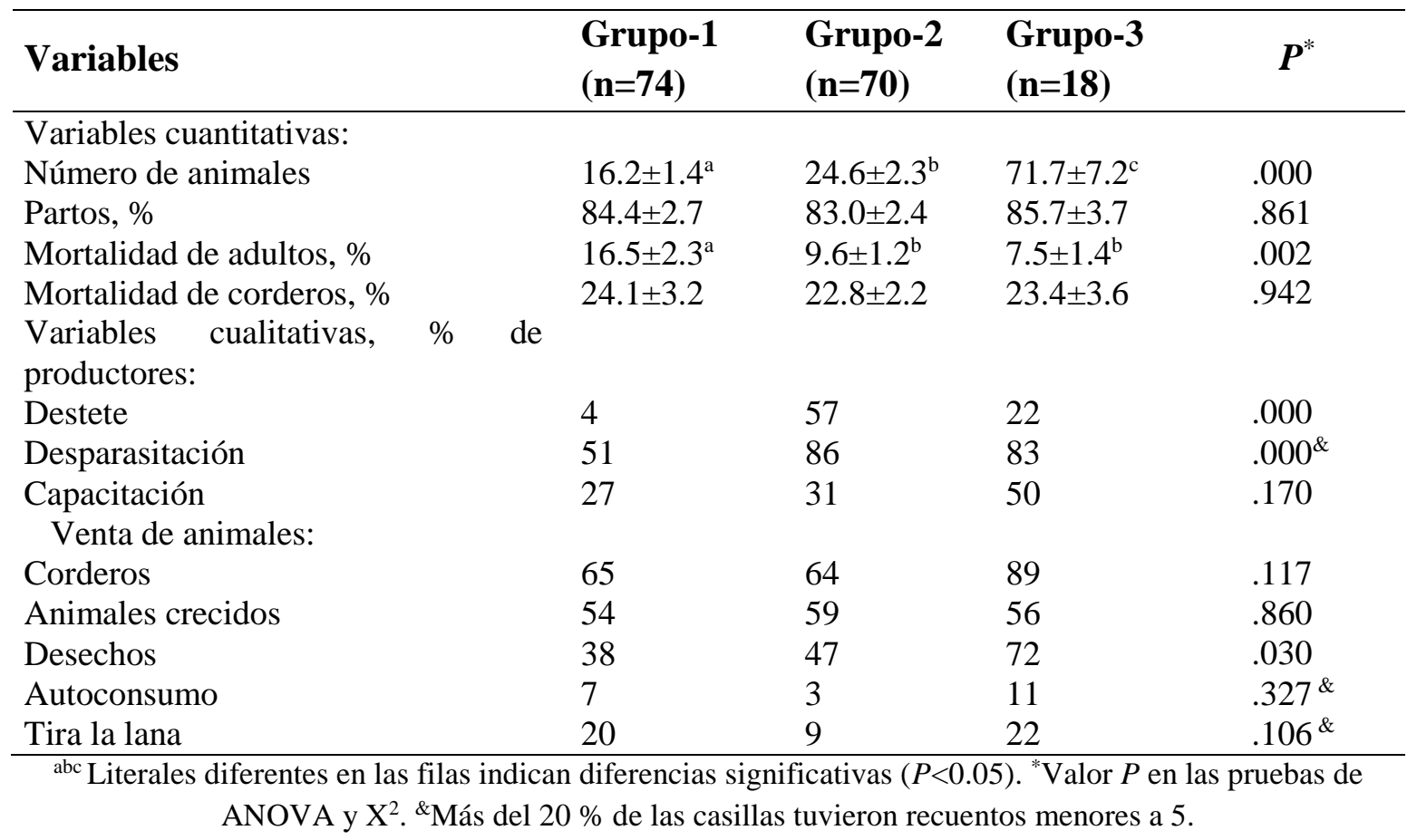

La comercialización de los productos ovinos se aprecia en el Cuadro 2. Los productores pequeños e intermedios venden algunos corderos de pocos meses de edad (de 4 a 5 ) y el resto a lo largo del año y menos de la mitad desecha animales improductivos. Esto confirma la seguridad financiera como una de las principales funciones de la ganadería, ya que los animales se comercializan cuando surgen necesidades económicas ${ }^{(18,23)}$. Por el contrario, los productores capitalizados venden más corderos y desechos, porque sus instalaciones y disponibilidad de mano de obra, no permiten aumentar la cantidad de animales. Este hallazgo sugiere que la dimensión de los rebaños se autorregula y la carga animal se mantiene estable, lo que puede conducir a mejorar la productividad del rebaño, sin aumentar su dimensión ${ }^{(15)}$, mediante la aplicación de prácticas técnicas de manejo reproductivo y tratamientos sanitarios. Respecto a la comercialización de lana, alrededor del $20 \%$ de los productores trasquilan sus borregos, pero desechan el producto debido "a su precio bajo" (\$1.00/Kg), por lo que el mercado limita el desarrollo de este sistema ${ }^{(14)}$.

\section{Producción agrícola}

Los pequeños productores cuentan con menor superficie agrícola, especies cultivadas y maquinaria, destinan mayor porcentaje de sus cosechas al autoconsumo, mientras un $12 \%$ no posee tierras de cultivo. Los productores intermedios tienen mayor diversidad de cultivos y uso de maquinaria, aunque ésta no es propia. Tienden a sembrar mayor porcentaje de avena 
forrajera (Avena sativa), haba (Vicia faba), chícharo (Pisum sativum) y frijol (Phaseolus vulgaris) $(P>0.05)$. Si bien, venden un porcentaje de papa (Solanum tuberosum) mayor que los pequeños productores, una tercera parte siembra cantidades marginales $(<0.3$ ha) para consumo familiar (Cuadro 3). Por otro lado, los productores capitalizados tienen más superficie cultivada y uso de maquinaria, cultivan papa (Solanum tuberosum) en el $18 \%$ de sus tierras y maíz (Zea mays) en el $26 \%$, mientras los otros grupos dedican el 9 y $41 \%$ de sus tierras, respectivamente. Los resultados indican que la proporción de especies cultivadas y la finalidad comercial de éstas, depende del nivel de capitalización de los productores.

En general, los tres grupos cultivan avena en el $50 \%$ de sus terrenos, destinan el maíz para autoconsumo y los forrajes y esquilmos para alimentar equinos y rumiantes, estos a su vez aportan abono para los cultivos, en un manejo complementario entre agricultura y ganadería, similar a otros $\mathrm{SPO}^{(18,22)}$. Estas evidencias destacan que los productores del NT han diseñado de manera tradicional, un uso integral de sus recursos.

Cuadro 3: Variables cuantitativas (media y EE) y cualitativas (\%) de producción agrícola en grupos de productores del Nevado de Toluca

\begin{tabular}{|c|c|c|c|c|}
\hline Variables & Grupo 1 & Grupo 2 & Grupo 3 & $P^{*}$ \\
\hline \multicolumn{5}{|l|}{ Variables cuantitativas: } \\
\hline Especies cultivadas & $1.9 \pm 0.1^{\mathrm{a}}$ & $2.5 \pm 0.1^{b}$ & $1.9 \pm 0.3^{\mathrm{ab}}$ & .004 \\
\hline Superficie cultivada, ha & $1.9 \pm 0.2^{\mathrm{a}}$ & $3.1 \pm 0.3^{\mathrm{a}}$ & $5.3 \pm 1.7^{\mathrm{b}}$ & .000 \\
\hline Maquinaria $\#$ & $1.9 \pm 0.2^{\mathrm{a}}$ & $3.3 \pm 0.2^{b}$ & $2.7 \pm 0.4^{\mathrm{ab}}$ & .000 \\
\hline \multicolumn{5}{|c|}{$\begin{array}{l}\text { Variables cualitativas, } \% \text { de } \\
\text { productores: }\end{array}$} \\
\hline \multicolumn{5}{|l|}{ Cultivos: } \\
\hline Avena (Avena sativa) & 70 & 86 & 83 & .069 \\
\hline Maíz (Zea mays) & 64 & 79 & 50 & .030 \\
\hline Papa (Solanum tuberosum) & 28 & 30 & 39 & .683 \\
\hline Otros: & 19 & 29 & 11 & .130 \\
\hline \multicolumn{5}{|l|}{ Autoconsumo del cultivo } \\
\hline Maiz (Zea mays) & 98 & 100 & 100 & $.503^{\mathrm{f}}$ \\
\hline Papa (Solanum tuberosum) & 52 & 29 & 14 & $.115^{\mathrm{f}}$ \\
\hline Otros & 86 & 81 & 50 & $.483^{\mathrm{f}}$ \\
\hline
\end{tabular}




\section{Aspectos socioeconómicos}

La familia (5 integrantes) de los pequeños productores tiene menor participación en actividades agropecuarias, porque al menos un miembro del hogar se inserta en empleos no agrícolas fuera del área protegida, lo que disminuye la dependencia de los recursos naturales ${ }^{(2)}$. Los productores intermedios tienen familias más grandes (6 personas) y con mayor participación en las actividades agropecuarias. No obstante, también tienen más fuentes de ingreso (Cuadro 4), porque diferentes miembros del hogar atienden al ganado, después de realizar otras actividades, (labores del hogar, empleos o estudios). Esta evidencia destaca la pluriactividad como una estrategia para aumentar los ingresos y asegurar el flujo de recursos financieros al hogar ${ }^{(10)}$. Los productores capitalizados tienen menor edad, terminaron la primaria y su familia de cinco integrantes, cubre al $100 \%$ el cuidado de los animales. Estos datos son consistentes con el sector rural mexicano ${ }^{(25)}$, pero contrastan con países europeos, donde el $30 \%$ de los productores tiene escolaridad media o alta ${ }^{(20)}$ y la familia aporta del 33 al $74 \%$ de la mano de obra ${ }^{(8,13)}$.

Cuadro 4: Aspectos socioeconómicos de los grupos de productores formados por el análisis cluster

\begin{tabular}{|c|c|c|c|c|}
\hline Variables & Grupo 1 & Grupo 2 & Grupo 3 & $\boldsymbol{P}^{*}$ \\
\hline $\begin{array}{l}\text { Edad jefe de } \\
\text { (años) }\end{array}$ & $52.6 \pm 1.6$ & $53.6 \pm 1.4$ & $48.7 \pm 2.4$ & .247 \\
\hline $\begin{array}{l}\text { Escolaridad del jefe de } \\
\text { familia (años) }\end{array}$ & $4.7 \pm 0.4$ & $3.8 \pm 0.3$ & $5.6 \pm 0.8$ & .061 \\
\hline Participación familiar ${ }^{\#}$ & $2.2 \pm 0.4^{\mathrm{a}}$ & $3.0 \pm 0.2^{\mathrm{b}}$ & $2.6 \pm 0.3^{\mathrm{ab}}$ & .004 \\
\hline Fuentes de ingreso & $5.3 \pm 0.2^{\mathrm{ab}}$ & $5.8 \pm 0.2^{\mathrm{b}}$ & $4.6 \pm 0.5^{\mathrm{a}}$ & .006 \\
\hline Ingreso ovino anual, \$ & $\begin{array}{l}5,699.2 \pm \\
790^{\mathrm{a}}\end{array}$ & $\begin{array}{l}17,554.0 \pm \\
4651^{\mathrm{a}}\end{array}$ & $\begin{array}{l}101,790.3 \pm \\
51257^{b}\end{array}$ & $0 \cap 0$ \\
\hline Aporte ovino al ingreso, $\%$ & $13.3 \pm 2.3^{\mathrm{a}}$ & $17.3 \pm 2.2^{\mathrm{a}}$ & $30.6 \pm 7.8^{b}$ & $\begin{array}{l}.000 \\
.009\end{array}$ \\
\hline Ingreso agrícola anual, \$ & $\begin{array}{l}11,123.5 \pm \\
3,051.4^{\mathrm{a}}\end{array}$ & $\begin{array}{l}33,291.9 \pm \\
12,218.2^{\mathrm{a}}\end{array}$ & $\begin{array}{l}133,943.3 \pm \\
70032.5^{\mathrm{b}}\end{array}$ & .001 \\
\hline
\end{tabular}

abc Diferentes literales en las filas indican diferencias significativas $(P<0.05) .{ }^{*}$ Valor $P$ en las pruebas de ANOVA. "Número de integrantes que participan en actividades agrícolas y ganaderas.

\section{Ingresos}

Los ingresos agrícolas y ganaderos fueron similares $(P>0.05)$ entre productores pequeños e intermedios (Cuadro 4). El análisis de varianza no detectó diferencias por la alta variabilidad dentro de grupos; este fenómeno se observa en estudios que integran aspectos socioeconómicos ${ }^{(6,13)}$, además de que el flujo de ingresos ovinos no es continuo, ya que los 
productores conservan animales que venden cuando necesitan dinero, de modo que hay ciclos productivos con ventas escasas y otros con ventas elevadas; por ejemplo, el $16 \%$ de los hogares no realizó ventas en el año de estudio. Los ingresos ovinos de los productores capitalizados fueron mayores, pero solo aportan la tercera parte de los ingresos totales del hogar, lo que indica que los SPO en el Nevado de Toluca son un complemento de la economía familiar $^{(14,24)}$, que se suma con empleo fuera del ANP, remesas y subsidios gubernamentales.

\section{Uso de los recursos de pastoreo}

El $97 \%$ de los productores pastorean su rebaño en la modalidad de pastoreo exclusivo y semi-estabulado (pastoreo-estabulación), de ellos el $58 \%$ ingresa al bosque, pero esta proporción aumenta en los productores capitalizados, quienes emplean en mayor porcentaje el pastoreo exclusivo, para no incrementar sus costos con la compra de alimentos ${ }^{(22)}$. Mientras que los productores pequeños e intermedios emplean el semi-estabulado (Cuadro 5), porque sus rebaños requieren menor volumen de alimento, esto les permite disminuir el pastoreo y canalizar su mano de obra en actividades de mayor ingreso ${ }^{(2)}$.

Cuadro 5: Pastoreo y manejo alimentario de los ovinos, por grupo de productores (\%)

\begin{tabular}{|c|c|c|c|c|}
\hline Variables & $\begin{array}{l}\text { Grupo } 1 \\
(n=74)\end{array}$ & $\begin{array}{l}\text { Grupo } 2 \\
(n=70)\end{array}$ & $\begin{array}{l}\text { Grupo } 3 \\
(n=18)\end{array}$ & $\boldsymbol{P}^{*}$ \\
\hline \multicolumn{5}{|l|}{ Manejo alimentario: } \\
\hline Pastoreo & 26 & 26 & 44 & .060 \\
\hline Semi-estabulado & 73 & 64 & 44 & .055 \\
\hline Estabulado & 1 & 10 & 11 & $.060^{\&}$ \\
\hline Pastoreo en bosque: & 57 & 51 & 67 & .490 \\
\hline \multicolumn{5}{|l|}{ Alimentos empleados: } \\
\hline Forraje henificado & 84 & 96 & 89 & .065 \\
\hline Alimentos balanceados & 18 & 31 & 39 & .068 \\
\hline Sal mineral & 64 & 84 & 100 & .001 \\
\hline
\end{tabular}

Los circuitos de pastoreo dentro del bosque son alternados con frecuencia y son diferentes entre productores, quienes modifican sus recorridos a lo largo del año, de acuerdo a su percepción de la disponibilidad de vegetación ${ }^{(5,9)}$. Estos hallazgos sugieren que la presión sobre los recursos de pastoreo, está regulada por la disponibilidad aparente de forraje, los ciclos de pastoreo anteriores y por las características agroecológicas in situ. Por lo que el manejo de los SPO puede ser compatible con los esfuerzos de conservación, si se ajusta la carga animal, mediante el desarrollo de metodologías adecuadas al pastoreo circulante y se realiza una adecuada transferencia de conocimientos entre los productores. 
Los rebaños se pastorean en el bosque principalmente en la época seca (marzo a mayo), cuando disminuye la cantidad y calidad de los pastos. En las lluvias (junio a octubre) se acude a los pastizales de los géneros Vulpia, Nassella, Trisetum, Muhlenbergia, Potentilla ${ }^{(26)}$ y disminuye el ingreso al bosque. Después de las cosechas (noviembre a febrero) se usan los rastrojos y las orillas de los caminos. Este manejo muestra que los SPO se adaptan a la disponibilidad de recursos a lo largo del año ${ }^{(22,26)}$ y la experiencia de los productores en el uso de áreas de pastoreo, puede emplearse para la zonificación en la nueva categoría del NT, integrando los conocimientos locales, al diseño de los planes de manejo ${ }^{(27)}$.

\section{Alimentación}

El $88 \%$ de los hogares suministra alimentos concentrados (comerciales y mezclas caseras elaboradas empíricamente) a su rebaño, lo que confirma que el manejo tradicional de pastoreo se está transformando en un sistema semi-estabulado, de pastoreo diurno con encierro y suplementación vespertina. Esto responde al interés por intensificar la producción $^{(1,5)}$ y a las estrategias de adaptación ante las restricciones en el uso y aprovechamiento de los recursos naturales ${ }^{(4)}$. La intensificación también se observó en la estabulación de corderos destetados (Cuadro 5), para engordarlos y obtener mejores precios de venta ( $22 \%$ de los productores). Sin embargo, como han señalado Pérez et $a l^{(3)}$, se debe cuidar que la intensificación no aumente los problemas de rentabilidad, debido a la compra de insumos.

La transición tecnológica observada en el NT se ha observado en diversos sistemas de producción $^{(5,7,14)}$ y sugiere que el uso de los recursos de pastoreo en ANP debería incorporarse a estrategias de alimentación más complejas, que incluyan alimentos comerciales y forrajes cultivados, sin dejar de considerar que los sistemas basados en pastoreo que integran adecuadamente sus recursos, pueden ser económicamente eficientes y ambientalmente amigables $^{(3,15)}$, ya que el pastoreo contribuye en mantener la biodiversidad $^{(10)}$ y evita la acumulación de material combustible ${ }^{(13,28)}$.

Los principales alimentos empleados en el NT, son avena henificada y rastrojo de maíz (Cuadro 5); estos tienen costos bajos por ser cultivos propios y subproductos. Pocos productores suministran mezclas con salvado de trigo o soya (13\%) o papa de desecho ( $2 \%)$, el uso de este recurso no se ha encontrado documentado. El manejo alimentario en el NT es similar a otros $\mathrm{SPO}^{(22,23)}$ y al manejo agrosilvopastoril tradicional descrito por Choocharoen $e a l^{(27)}$, donde los recursos se usan de manera integrada, aunque los diferentes estratos vegetales, no necesariamente comparten el mismo espacio. Esto difiere de los diseños agroecológicos que han analizado algunos investigadores como Cubbage et al ${ }^{(28)}$, en los que se combinan árboles con diferentes estratos de vegetación, para la alimentación animal. 


\section{Conclusiones e implicaciones}

Se identifican tres grupos de productores, diferenciados por el nivel de capitalización observado en la cantidad de animales, terrenos que poseen y los ingresos que generan. Sin embargo, la capitalización no se refleja en la productividad, ya que los tres grupos tienen resultados similares en la tasa de pariciones y mortalidad de corderos, por lo tanto, se requiere capacitación que permita elevar la producción y los ingresos de la región. La producción agrícola sí se correlaciona con la capitalización de los productores, pues mientras más activos productivos poseen, se dedica mayor superficie a cultivos comerciales. La relación cultivos - ganado se alinea con los esfuerzos de conservación, ya que el uso de estiércoles disminuye la aplicación de abonos de síntesis química. Los aspectos sociales, como edad y escolaridad no inciden en la productividad del sistema ovino, pero su eficiencia económica depende de la mano de obra familiar. La producción ovina no es la fuente principal de ingresos, pero es esencial en la economía familiar porque es un factor de seguridad financiera, que ningún otro componente económico puede suplir. Cualquier plan de manejo para la zona debería considerar que, el sistema de producción ovina está en transición de pastoreo extensivo a semi-estabulado, por lo que requiere mejorar las estrategias de alimentación y con esto disminuir el pastoreo dentro del área protegida, o en su caso definir planes de acción para un aprovechamiento sustentable, basados en el nivel de deterioro de las zonas de pastoreo y su relación con la carga animal. La adopción de un manejo agrosilvopastoril puede hacer compatible la producción ovina con los esfuerzos de conservación. Sin embargo, por las características biológicas y socioeconómicas de la zona, para lograr un modelo técnicamente viable se requiere la interacción y trabajo concurrente de los múltiples actores con injerencia e intereses dentro del ANP.

\section{Agradecimientos}

Este trabajo se realizó con el financiamiento de la Universidad Autónoma del Estado de México, a través del proyecto "Ganadería Modos de vida y paisaje del Área de Protección de Flora y Fauna Nevado de Toluca. Interacciones y co-evolución desde inicios del siglo XX”. Se agradece a los productores del Nevado de Toluca, por su confianza y la disponibilidad de tiempo para la realización del estudio. 


\section{Literatura citada:}

1. Herrero M, Thornton PK, Gerber P, Reid RS. Livestock, livelihoods and environmental: understanding the trade-offs. Current Opinion in Environmental Sustainability 2009;(1):111-120.

2. Babulo B, Muys B, Nega F, Tollens E, Nyssen J, Deckers J, et al. Household livelihood strategies and forest dependence in the highlands of Tigray, Northern Ethiopia. Agric Syst 2008;(98):147-155.

3. Pérez JP, Gil JM, Sierra I. Technical efficiency of meat sheep production systems in Spain. Technical note. Small Ruminant Res 2007;(69):237-241.

4. Gurung B, Nelson KC, Smith JLD. Impact of grazing restrictions on livestock composition and husbandry practices in Madi Valley, Chitwan National Park, Nepal. Environmental Conservation 2010;36(4):338-347.

5. Jouven M, Lapeyronie P, Moulin CH, Bocquier F. Rangeland utilization in Mediterranean farming systems. Animal 2010;4(10):1746-1757.

6. Madry W, Mena Y, Roszkowska-Madra B, Gozdowski D, Hryniewski R, Castel JM. An overview of farming system typology methodologies and its use in the study of pasturebased farming system: a review. Spanish J Agric Res 2013;11(2):316-326.

7. Toro-Mujica P, Aguilar C, Vera R, Rivas J, García A. Sheep production systems in the semi-arid zone: Changes and simulated bio-economic performances in a case study in Central Chile. Livestock Sci 2015;(180):209-219.

8. Gaspar P, Escribano M, Mesías FJ, Rodriguez LA, Pulido F. Sheep farms in the Spanish rangelands (dehesas): Typologies according to livestock management and economic indicators. Small Ruminant Res 2008;(74):52-63.

9. Lasseur J. Sheep farming systems and nature management of rangeland in French Mediterranean mountain areas. Livest Prod Sci 2005;(96):87-95.

10. Riedel L, Casasús I, Bernués A. Sheep farming intensification and utilization of natural resources in a Mediterranean pastoral agro-ecosystem. Livestock Sci 2007;(111):153163.

11. CONANP. Comisión Nacional de Áreas Naturales Protegidas. Estudio Previo Justificativo para la Modificación de la Declaratoria del Parque Nacional Nevado de Toluca, ubicada en el Estado de México, México. 2013. 
12. DOF. Diario Oficial de la Federación. Decreto que reforma, deroga y adiciona diversas disposiciones del diverso publicado el 25 de enero de 1936, por el que se declaró Parque Nacional la montaña denominada "Nevado de Toluca". 2013. http://www.nevadodetoluca.conanp.gob.mx/decreto.php\#.WAefVP196M8. Consultado: 19 Oct, 2015.

13. Toro-Mujica P, García A, Gómez-Castro A, Perea J, Rodríguez-Estévez V, Angón E. et al. Organic dairy sheep farms in south-central Spain: Typologies according to livestock management and economic variables. Small Ruminant Res 2012;(104):28-36.

14. Raineri C, Nunes BCP, Gameiro AH. Technological characterization of sheep production systems in Brazil. Anim Sci J 2015;(86):476-485.

15. Al-Khalidi KM, Alassaf AA, Al-Shudiefat MF, Al-Tabini RJ. Economic performance of small ruminant production in a protected area: a case study from Tell Ar-Rumman, a Mediterranean ecosystem in Jordan. Agric Food Econom 2013;1:8.

16. Hair JF, Black WC, Tatham RL, Anderson RE. Multivariate Data Analysis. 7th ed. London, United Kingdom. Prentice Hall International; 2010.

17. Field, A. Discovering statistics using IBM SPSS statistics. 4th ed. Great Britain: SAGE Publications; 2013.

18. Vázquez MI, Vargas LS, Zaragoza RJL, Bustamante GA, Calderón SF, Rojas AJ, et al. Tipología de explotaciones ovinas en la sierra norte del estado de Puebla. Téc Pecu Méx 2009;47(4):357-369.

19. Cortez-Arriola J, Rossing AHW, Améndola MRD, Scholberg MSJ, Groot JCJ, Tittonell P. Leverages for on-farm innovation from farm typologies? An illustration for familybased dairy farms in north-west Michoacán, Mexico. Agric Syst 2015;(135):66-76.

20. Rivas J, García A, Toro-Mujica P, Angón E, Perea J, Morantes M, et al. Caracterización técnica, social y comercial de las explotaciones ovinas manchegas, centro-sur de España. Rev Mex Cienc Pecu 2014;5(3):291-306.

21. Mier y Terán M, Rabell C. Escolaridad y lengua hablada en comunidades rurales de la península yucateca. Rev Mex Sociología 2013;75(3):371-406. 
22. Galaviz-Rodríguez JR, Vargas-López S, Zaragoza-Ramírez JL, Bustamante-González A, Ramírez-Bribiesca E, Guerrero-Rodríguez JD, et al. Evaluación territorial de los sistemas de producción ovina en la región nor-poniente de Tlaxcala. Rev Mex Cienc Pecu 2011;2(1):53-68.

23. Kosgey IS, Rowlands GJ, Van-Arendonk JAM, Baker RL. Small ruminant production in smallholder and pastoral/extensive farming systems in Kenya. Small Ruminant Res 2008;(77):11-24.

24. Pérez HP, Vilaboa AJ, Chalate MH, Martínez BC, Díaz RP, López OS. Análisis descriptivo de los sistemas de producción con ovinos en el estado de Veracruz, México. Rev Científica 2011;XXI(4):327-334.

25. Ordaz JL. México: capital humano e ingresos. Retornos a la educación, 1994-2005. En: CEPAL - Serie Estudios y perspectivas - México. 2007;(90).

26. Martínez HJ, Arriaga JCM, González RIC, Rosa GR, Hernández LGB, Valdés RJ, et al. La acumulación neta de fitomasa y calidad nutritiva de pastizales en el Área de Protección de Flora y Fauna Nevado de Toluca para la producción ovina. Reunión Científica de la Sociedad Española para el Estudio de los Pastos. Lugo-A Coruña, España 2016:381-386.

27. Choocharoen C, Neef A, Preechapanya P, Hoffmann V. Agrosilvopastoral Systems in Northern Thailand and Northern Laos: Minority Peoples' Knowledge versus Government Policy. Land 2014;(3):414-436.

28. Cubbage F, Balmelli G, Bussoni A, Noellemeyer E, Pachas AN. Fassola H, et al. Comparing silvopastoral systems and prospects in eight regions of the world. Agroforest Syst 2012;(86):303-314. 